\title{
The origin of molecular protostellar outflows
}

\author{
I. Contopoulos ${ }^{1, \star}$ and C. Sauty ${ }^{2}$ \\ 1 Physics Department, University of Crete, PO Box 2208, Heraklion 71003, Greece \\ e-mail: jkontop@elval.vionet.gr \\ 2 Observatoire de Paris, Université de Paris 7, DAEC, 92190 Meudon, Paris, France \\ e-mail: christophe.sauty@obspm.fr
}

Received 11 August 2000 / Accepted 4 October 2000

\begin{abstract}
We study the geometry of the large scale magnetic field threading the centrifugally supported circumstellar disk that forms in the inner few hundred AU of a dynamically collapsing protostellar molecular cloud core. The disk is highly turbulent, therefore highly diffusive, thus unable by itself to sufficiently stretch and bend the large scale magnetic field so as to launch magnetically driven winds from its surface. However, when we take proper account of the fact that the magnetic field is already highly stretched and bent in the outside dynamically collapsing cloud core, we discover that this contributes to significant outward magnetic field bending around the outer edge of the inner protoplanetary disk too. We obtain the field geometry self-consistently under general simplifying approximations. We also show how this magnetic field geometry leads naturally to strong magneto-centrifugally driven outflows from the surface of the disk, with mass loss rates a significant fraction of the accretion rate onto the inner disk. Our simple picture describes naturally the origin of the molecular winds observed during the end stages of protostellar cloud collapse.
\end{abstract}

Key words. MHD - stars: winds, outflows - ISM: jets and outflows

\section{Introduction}

Low mass stars form in the gravitational collapse of massive interstellar molecular cloud cores. The details of the collapse have been studied extensively during the past couple of decades, and a coherent modern theory of star formation slowly begins to emerge. The early phase of collapse before a protostar begins to form at the very center (i.e. the first few $10^{7}$ years) is believed to be well understood (see Mouschovias 1996 for a review). Only very recently have people begun to investigate how the collapse evolves after a central protostar forms, and several surprises awaited us, having to do with the formation of various "expansion fronts". Before we proceed, let us briefly sketch what we believe are the main physical stages which lead to the formation of a normal star surrounded by a protoplanetary disk.

Thermally supercritical dense interstellar molecular clouds with masses in the range $10^{2}-10^{5} M_{\odot}$, neutral particle number densities around $10^{3} \mathrm{~cm}^{-3}$, specific angular momentum of the order $10^{22} \mathrm{~cm}^{2} \mathrm{~s}^{-1}$, and temperatures around $10 \mathrm{~K}$, are held from collapsing dynamically under their own weight by the pressure and tension of ordered magnetic fields which thread them over

Send offprint requests to: I. Contopoulos

* Present address: 200 Akti Themistokleous Str.,

Piraeus 18539, Greece. large scales (Crutcher et al. 1996; Crutcher 1998). Quoting Mouschovias (1996), "the presence of the magnetic field does not prevent the collapse, it simply regulates it, that is, it slows down the contraction through ambipolar diffusion drag, and converts the would-be violent free fall into a quasi-static process". The inner portions of the cloud are loaded with more and more neutral matter which slowly diffuses through the magnetic field lines. During that stage, magnetic braking decreases the specific angular momentum by more than two orders of magnitude (Basu \& Mouschovias 1995a; Basu \& Mouschovias 1995b; Basu 1997; Ciolek \& Basu 2000), to a value comparable to that of the protosolar nebula (Hayashi et al. 1993; Ohasi et al. 1996; Saito et al. 1996; Ohashi et al. 1997). It is very interesting that, at the late stages of core collapse, a self-similar profile develops, with a constant ratio of local specific angular momentum $j$ to total mass $m$ enclosed inside (Basu 1997; Saigo \& Hanawa 1998; Nakamura et al. 1999),

$\frac{j}{m}=\omega \frac{G}{c_{\mathrm{s}}}$

with $0<\omega<0.5$ a very important (as we will see) parameter which is determined by the background large scale magnetic field and cloud rotation (Basu 1998 gives a value of order 0.026$) ; c_{\mathrm{S}} \equiv\left(k_{\mathrm{B}} T / \mu\right)^{1 / 2}$ is the isothermal speed of sound; $\mu=2.33 m_{\mathrm{H}}$ is the mean particle weight of a fluid consisting of molecular hydrogen with a $10 \%$ [by number] 
helium admixture. This first stage of the cloud's evolution lasts for about $10^{6}-10^{7}$ years (Ciolek \& Basu 2000; Ciolek \& Basu 2001). When an inner magnetically supercritical core forms, it begins to collapse more and more dynamically, forming an inward increasing density profile with an ever decreasing (in size and mass) inner region of higher and higher density, and a similar highly centrally concentrated distribution of magnetic field. Angular momentum removal through magnetic torques proceeds slower than the dynamic collapse, and angular momentum is effectively conserved. At the end of this second dynamical stage of the cloud's evolution which lasts for about another $10^{6}$ years (Ciolek \& Mouschovias 1995), the central accretion proceeds at a rate of about 1 to 10 solar masses per million years (i.e. a few times the "canonical" value $\left.c_{\mathrm{s}}^{3} / G\right)$.

What happens after that time, only recently have we begun to understand, by treating the inner part of the collapse as a mathematical sink of matter and magnetic flux (Larson 1969; Penston 1969; Shu 1977; Hunter 1977; Foster \& Chevalier 1993; Li 1998a; Li 1998b; Ciolek \& Königl 1998, hereafter CK; Contopoulos et al. 1998, hereafter CCK). As the core collapse proceeds and matter enters into the center, the central gravity dominates over the self-gravity of the molecular disk. As a result, the inner part begins to collapse in free fall, under the action of the gravitational pull of the central growing mass, "diluted" by the pull of the outward tension of the magnetic field which is carried along with the inflow (Shu \& Li 1997).

"Diluted" free-fall cannot proceed all the way to the origin. The reason is simple: magnetic field tension leads to high outward magnetic field diffusion, and the field eventually stays in place, i.e. it becomes effectively decoupled from the neutral material, which continues to collapse in "undiluted" free-fall. The region where magnetic flux piles up grows with time, and a magnetic front propagates outward in the form of a C-shock (see CK, CCK for a detailed presentation; Li \& McKee 1996, Li 1998a and Li 1998b treat the same problem ignoring magnetic field tension).

Rotation does not significantly inhibit free-fall down to a distance of about $j^{2} /(G m)$. The flow will be abruptly decelerated in a strong hydrodynamic shock at

$$
\begin{aligned}
r_{\text {shock }} & \approx \frac{\omega^{2} G m_{\mathrm{d}}}{c_{\mathrm{s}}^{2}} \\
& =250 \mathrm{AU}\left(\frac{m_{\mathrm{d}}}{M_{\odot}}\right)\left(\frac{T}{10 \mathrm{~K}}\right)^{-1}\left(\frac{\omega}{0.1}\right)^{2},
\end{aligned}
$$

inside which a centrifugally supported disk can form. As the mass $m_{\mathrm{d}}$ of the disk + forming protostar grows, the shock will move outwards at a rate

$$
\begin{aligned}
\dot{r}_{\text {shock }}= & 10^{-3} \mathrm{~km} \mathrm{~s}^{-1} \\
& \times\left(\frac{\dot{m}_{\mathrm{accr}}}{10^{-6} M_{\odot} \mathrm{yr}^{-1}}\right)\left(\frac{T}{10 \mathrm{~K}}\right)^{-1}\left(\frac{\omega}{0.1}\right)^{2} .
\end{aligned}
$$

This is shown clearly in the work of Saigo \& Hanawa (1998), who studied the hydrodynamic collapse of a rotating core under idealized conditions of self-similarity.
We take as a well known fact (Dubrulle 1992; Balbus \& Hawley 1991) that this differentially rotating disk will be turbulent, therefore also highly viscous and diffusive ${ }^{1}$. In other words, the magnetic Prandtl number $\mathcal{P}_{\mathrm{m}} \equiv \eta / \nu$ has its nominal value of order unity (Bardou \& Heyvaerts 1996; Lubow et al. 1994). Here, $\eta$ is the effective turbulent magnetic diffusivity; and $\nu$ is the kinematic shear viscosity.

We take off at this point to speculate what happens after such a centrifugally supported disk forms. In Sects. 2 and 3, we investigate what the above implies for the structure of the large scale magnetic field which is known to be carried inward in the early collapse of the magnetically supercritical molecular cloud core. In Sect. 4, we study the distribution of matter outflow from the surface of the disk, making the working assumption that the structure of magnetic field obtained before is "infinitely rigid" above the surface of the disk. We thus discover that the rate of mass loss in the wind can be a significant fraction (up to $100 \%$ ) of the rate of mass accretion in the freely-falling collapsing core! Our conclusions, summarized in Sect. 5, are that molecular outflows may appear in the very early stages of proplanetary disk formation, originating from the region around the edge of the protostellar disk, and not necessarily from an extended region along the disk. The above seem to account very naturally for the formation of class 0 molecular outflows with velocities of the order of a few tens of kilometers per second ${ }^{2}$ and for their formation before any fast optical jets appear to emerge from the center (André et al. 1993).

\section{The magnetic field}

The inflow will be highly compressed along its equatorial plane by gravitational tidal forces (both in the exterior freely-falling molecular cloud core, and in the interior centrifugally supported viscous disk). Vertical

\footnotetext{
${ }^{1}$ Rotating hydrodynamic flows where $\mathrm{d} j / \mathrm{d} r>0$ (as is the case in the above disks) are known to be linearly stable (Rayleigh 1916). Here, $j \equiv r v_{\phi}$. This is also corroborated by the low Reynolds number numerical simulations of Balbus et al. (1996). However, the linear analysis does not take into account the contribution of finite amplitude (i.e. nonlinear) perturbations, which are well known to lead to significant turbulence in laboratory flows with high Reynolds numbers (e.g. Wendt 1933; Taylor 1936a; Taylor 1936b; see Zahn 1990 for a review). This regime has begun to be studied only very recently in numerical simulations (Zahn, personal communication; Richard \& Zahn 1999). An independent school of thought points to the magnetic shearing instability in the presence of a weak magnetic field (Chandrasekhar 1960; Chandrasekhar 1981; Balbus \& Hawley 1991) as the origin of the elusive disk turbulence (e.g. Brandenburg et al. 1996).

${ }^{2}$ It is well known from the theory of MHD winds that the asymptotic outflow velocity is comparable to the Keplerian velocity of rotation at the base of the outflow.
} 
hydrostatic equilibrium implies that the disk has a thermal scale height ${ }^{3}$

$$
\begin{aligned}
h(r)= & \frac{c_{\mathrm{s}} r^{3 / 2}}{(G m)^{1 / 2}} \\
= & 25 \mathrm{AU}\left(\frac{r}{r_{\text {shock }}}\right)^{3 / 2}\left(\frac{m}{M_{\odot}}\right)^{-1 / 2}\left(\frac{m_{\mathrm{d}}}{M_{\odot}}\right)^{3 / 2} \\
& \times\left(\frac{T}{10 \mathrm{~K}}\right)^{-1}\left(\frac{\omega}{0.1}\right)^{3},
\end{aligned}
$$

where again $m$ is the mass contained inside radius $r$. Outside $r_{\text {shock }}, m \approx m_{\mathrm{d}}$, since, to a good approximation, the mass is concentrated in the central disk + forming protostar. As a first step, we will assume that the mass inside $r_{\text {shock }}$ is initially distributed as $m=m_{\mathrm{d}} r / r_{\text {shock }}$ (Eq. (3), Saigo \& Hanawa 1998), although a subsequent redistribution due to viscous torques is expected (Basu 1998). The exact mechanism of mass and angular momentum redistribution remain uncertain, thus we prefer at this point to consider the Keplerian disk at its very initial stage before any accretion process has taken place.

We will now study the magnetic field structure in the interior of the centrifugally supported disk $\left(r<r_{\text {shock }}\right.$ and $z \leq h(r)$ ), assuming that the flow has reached a steadystate where magnetic field lines are held in place, and no magnetic flux is advected inward (or outward). Balancing flux advection with diffusion in the disk interior yields

$\eta\left(\frac{\partial B_{z}}{\partial r}-\frac{\partial B_{\mathrm{r}}}{\partial z}\right)=v_{\mathrm{r}} B_{z} \approx 0$,

hence we obtain the simple result that

$\Delta^{*} \Psi \approx 0$

inside the centrifugally supported disk, where free-fall accretion has stopped $\left(v_{\mathrm{r}} \approx 0\right)$. Here, $\Delta^{*} \equiv\left(\partial^{2} / \partial r^{2}-\right.$ $\left.r^{-1} \partial / \partial r+\partial^{2} / \partial z^{2}\right)$ is the usual linear elliptic operator which appears in magnetostatic problems; and the magnetic flux function $\Psi$ is defined such that $B_{z}=$ $(1 / r) \partial \Psi / \partial r$, and $B_{\mathrm{r}}=-(1 / r) \partial \Psi / \partial z$.

Outside $r_{\text {shock }}$ things are more complicated. At those distances, the flow has a significant (almost free-fall) accretion velocity, and compression due to the in-falling neutrals works very effectively to highly stretch and distort the magnetic field. Since the pioneering analysis of Li \& McKee (1996) (see also CK, CCK for more details), we know that behind the expanding magnetic $\mathrm{C}$-shock, ions are held in place and neutrals accrete through them in free-fall, i.e. $\mathbf{v}_{i} \approx 0$. At those distances, ambipolar diffusion friction of freely-falling neutrals with non-accreting ions imparts a strong inward force to the ions, which is balanced by an outward magnetic force resulting from

\footnotetext{
3 As we will see, the magnetic field develops a non zero horizontal component $B_{\mathrm{r}} \neq 0$ above the disk, and thus, the associated magnetic pressure $B_{\mathrm{r}}^{2} /(8 \pi)$ also contributes to compress the disk vertically. We have verified (a posteriori) that this contribution is minor, and refer the reader to Eq. (9) of Ciolek \& Königl (1998) for the full expression for the disk scale height.
}

the stretching of the magnetic field tied onto them. This implies,

$$
\frac{1}{c} \mathbf{J} \times \mathbf{B}=\frac{\rho_{\text {disk }} \mathbf{v}_{\mathrm{D}}}{\tau_{n i}} .
$$

Here, $\rho_{\text {disk }} \equiv \dot{m}_{\text {accr }} /\left[4 \pi r h\left|v_{\mathrm{r}}\right|\right]$ is the average disk density, and

$$
\begin{aligned}
\mathbf{v}_{\mathrm{D}} & \equiv \mathbf{v}_{i}-\mathbf{v}_{n} \approx-\mathbf{v}_{n} \equiv-v_{\mathrm{r}} \hat{\mathbf{r}} \\
& =\left(\frac{G m_{\mathrm{d}}}{r}\right)^{1 / 2}\left(2-\frac{r_{\text {shock }}}{r}\right)^{1 / 2} \hat{\mathbf{r}}
\end{aligned}
$$

is the drift velocity of ions with respect to neutrals (the extra factor at the rhs takes rotation with angular momentum conservation into account); $\tau_{n i}=1.4\left(m_{i}+\right.$ $\left.m_{\mathrm{H}_{2}}\right) /\left[\rho_{i}<\sigma w>_{i \mathrm{H}_{2}}\right]$ is the neutral-ion mean collision time $\left(m_{i}=29 m_{\mathrm{H}}, m_{\mathrm{H}_{2}}\right.$ are the masses of a typical ion, and a hydrogen molecule respectively; the factor 1.4 takes into account the fact that the molecular hydrogen gas has a $10 \%$ helium number density admixture; $\langle\sigma w\rangle_{i \mathrm{H}_{2}}=1.6910^{-9} \mathrm{~cm}^{3} \mathrm{~s}^{-1}$ is the average collisional rate between ions and hydrogen molecules (e.g. McDaniel \& Mason 1973)). By balancing ionization by cosmic rays with dissociative recombination, we express the ion density as $\rho_{i} \approx m_{i}\left(\xi \rho_{\text {disk }} / \alpha_{\text {dr }} \mu\right)^{1 / 2}$ (Elmegreen 1979), where $\xi=10^{-17} \mathrm{~s}^{-1}$ is the cosmic ray ionization rate, and $\alpha_{\mathrm{dr}} \approx 10^{-6} \mathrm{~cm}^{3} \mathrm{~s}^{-1}$ is the electron dissociative recombination rate (e.g. Dalgarno 1987). Noting that $(1 / c) \mathbf{J} \times\left.\mathbf{B}\right|_{r}=-\Delta^{*} \Psi \cdot B_{z} /(4 \pi r)$, Eq. (7) implies

$$
\begin{aligned}
\frac{1}{B_{\text {eq }}} \Delta^{*} \Psi= & -\delta\left(2-\frac{r_{\text {shock }}}{r}\right)^{-1 / 4}\left(\frac{r}{r_{\text {shock }}}\right)^{-5 / 2} \\
& \times\left(\frac{B_{z}}{B_{\text {eq }}}\right)^{-1}
\end{aligned}
$$

with

$$
\begin{aligned}
\delta= & \frac{m_{i}<\sigma w>_{i \mathrm{H}_{2}} \xi^{1 / 2} \dot{m}_{\mathrm{accr}}^{1 / 2}}{4 \pi^{1 / 2} 1.4\left(m_{i}+m_{\mathrm{H}_{2}}\right) \alpha_{\mathrm{dr}}^{1 / 2} \mu^{1 / 2} c_{\mathrm{s}}^{3 / 2} \omega} \\
\equiv & 8\left(\frac{\dot{m}_{\mathrm{accr}}}{10^{-6} M_{\odot} \mathrm{yr}^{-1}}\right)^{1 / 2}\left(\frac{T}{10 \mathrm{~K}}\right)^{-3 / 4}\left(\frac{m_{\mathrm{d}}}{M_{\odot}}\right)^{-3 / 2} \\
& \times\left(\frac{\omega}{0.1}\right)^{-1}\left(\frac{\xi}{10^{-17} \mathrm{~s}^{-1}}\right)^{1 / 2}
\end{aligned}
$$

inside the freely-falling outside core at $r>r_{\text {shock}}$. Here,

$$
\begin{aligned}
B_{\mathrm{eq}}= & \frac{2^{1 / 2} \dot{m}^{1 / 2} c_{\mathrm{s}}^{5 / 2}}{m_{\mathrm{d}} \omega^{2} G} \equiv 0.4 \mathrm{mG}\left(\frac{\dot{m}_{\mathrm{accr}}}{10^{-6} M_{\odot} \mathrm{yr}^{-1}}\right)^{1 / 2} \\
& \times\left(\frac{T}{10 \mathrm{~K}}\right)^{5 / 4}\left(\frac{m_{\mathrm{d}}}{M_{\odot}}\right)^{-1}\left(\frac{\omega}{0.1}\right)^{-2}
\end{aligned}
$$

is the magnetic field value in equipartition with gas pressure at $r=r_{\text {shock }}$.

Outside the disk $(z>h(r))$ we will assume the simplest possible magnetic field configuration, namely one where electric currents and stresses are absent, i.e. current-free force-free $\left(B_{\phi}=0\right.$ and $\left.\Delta^{*} \Psi=0\right)$. Under certain conditions that we will study in the next section, 


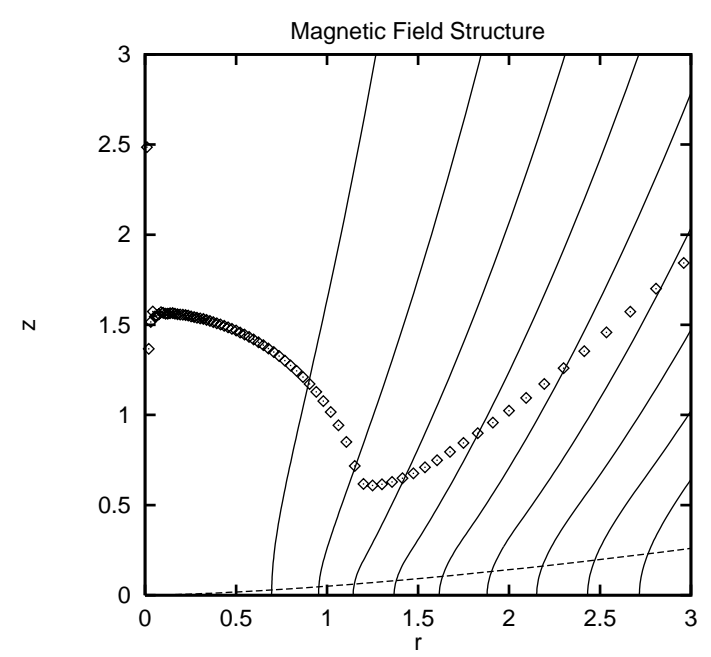

Fig. 1. Magnetic field geometry for $\omega=0.14$ and $\delta=5.7$. We plot here the magnetic field lines that correspond to values of $\Psi=n \times 0.2 B_{\text {eq }} r_{\text {shock }}^{2}$, where $n=1,2,3 \ldots$ The units of radial distance $r$ and vertical distance $z$ are $r_{\text {shock }}$. For the present choice of parameters, $B_{\text {eq }}=0.2 \mathrm{mG}$, and $r_{\text {shock }}=490 \mathrm{AU}$. We also plot the surface of the disk (dashed line), and the sonic surface of the wind outflow (squares)

matter is naturally launched above the disk surface and flows in the magnetosphere. At the places where this outflow is accelerated to very high velocities, the matter inertia will modify the geometry of the outside magnetosphere. However, near the origin of the outflow, i.e. in the region where the flow is not yet accelerated to Alfvénic velocities, the force-free assumption can yield the magnetic field geometry to a fair approximation. As we will see, in order to study the physical conditions for the launching of thermally + magnetically driven matter outflows from the surface of the disk, we are indeed interested only in the magnetic field structure near the surface of the disk, and not in its overall large scale distribution.

\section{Numerical procedure}

Let us now proceed with the numerical solution for the magnetic field structure, inside and outside our disk, rescaling lengths to $r_{\text {shock}}$, and magnetic fields to $B_{\text {eq }}$. Defining $R \equiv r / r_{\text {shock }}, Z \equiv z / r_{\text {shock }}, b_{z} \equiv B_{z} / B_{\text {eq }}$, and $\psi \equiv \Psi r_{\text {shock }}^{2} / B_{\text {eq }}$, we obtain

$$
\begin{aligned}
\Delta^{*} \psi= & -\frac{\delta}{R^{2.5} b_{z}}\left[2-\frac{1}{R}\right]^{-1 / 4} \\
& \text { for } R \geq 1 \text { and } Z \leq h(r) / r_{\text {shock }}, \text { and } \\
= & 0 \text { otherwise },
\end{aligned}
$$

and the boundary conditions

$$
\begin{aligned}
\frac{\partial \psi}{\partial R} & =0 \text { along } Z=0, \text { and } \\
\psi & =0 \text { along } R=0
\end{aligned}
$$

(the former boundary condition implies the dipolar overall magnetic field geometry with field lines crossing the equatorial disk mid-plane vertically).

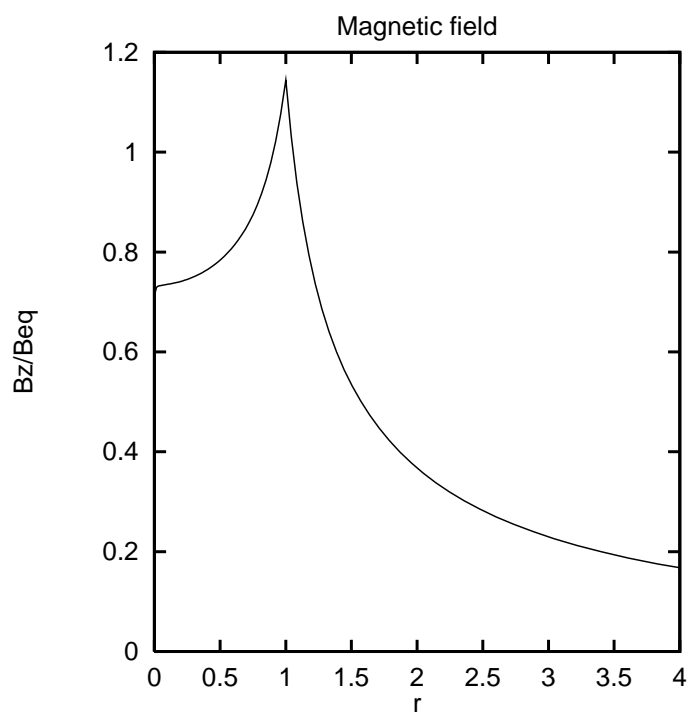

Fig. 2. The distribution of the magnetic field in units of $B_{\text {eq }}$ at the mid-plane of the disk, as a function of cylindrical radius along the disk in the solution of Fig. 1 (for our choice of parameters, $B_{\text {eq }}=0.2 \mathrm{mG}$ )

Our numerical procedure is to rescale our equation to the new variables $x \equiv R /(R+1)$, and $y \equiv Z /(Z+1)$. That way, solving the problem numerically in the interval $[0,1] \times[0,1]$ gives us the solution in all physical space, which means that we do not have to worry about what boundary conditions we implement at infinity ${ }^{4}$. We have used the elliptic solver provided in Numerical Recipes (Press et al. 1988). This implements the simultaneous over-relaxation solution of a linear (i.e. with coefficients fixed in space) elliptic equation with Chebyshev acceleration, in a rectangular computational grid. Our present problem is clearly non-linear. One can, however, regard the right-hand side of Eq. (11) as a function of position at each iteration step, after making an initial guess for $\psi(x, y)$. The iteration procedure is repeated until the input and output $\psi$ 's differ by less than $10^{-4}$ of their values. In a grid of $100 \times 100$ points, we reach convergence in about $10^{5}$ iterations.

The solution of our numerical integration for $\omega=0.14$ and $\delta=5.7$ is shown in Fig. 1. We plot here magnetic field lines that correspond to values of $\psi=n \times 0.2$, where $n=$ $1,2,3 \ldots$ (we remind the reader that $\psi=1$ corresponds to magnetic flux $\left.\Psi=B_{\text {eq }} r_{\text {shock }}^{2}\right)$. We also plot the surface of the disk (Eq. (4)), and the sonic surface of the wind outflow (see Sect. 4). The distribution of the magnetic field in units of $B_{\text {eq }}$ at the mid-plane of the disk is shown in Fig. 2 (we again remind the reader that for our choice of parameters, $B_{\text {eq }}=0.2 \mathrm{mG}$ ).

The first thing one observes is that, although no significant force is acting on the magnetic field in the inner protoplanetary disk, the magnetic field geometry is highly distorted at the surface of the disk because of the high

4 The reader should contrast this approach with the finite spatial extent solvers of Lubow et al. (1994); Reyes-Ruiz \& Stepinski (1996); see also Sect. 5. 


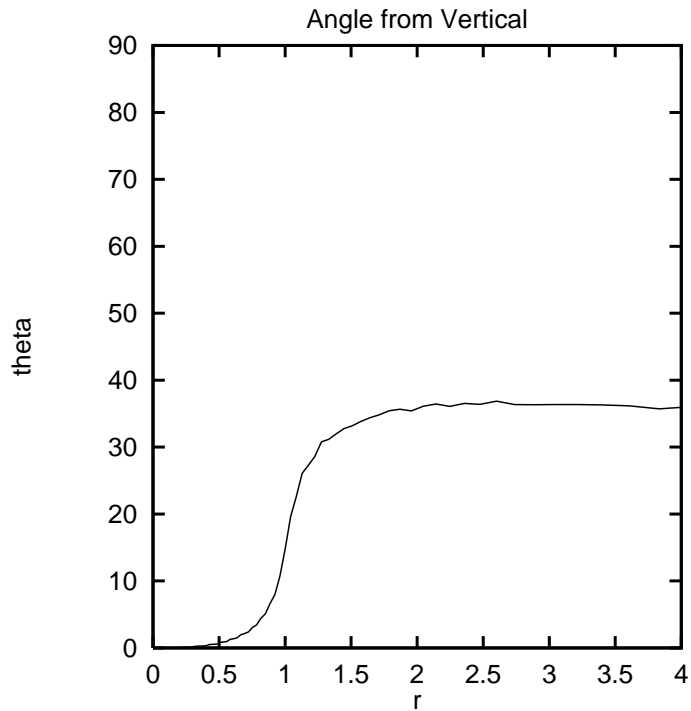

Fig. 3. The distribution of the field bending angle $\theta$ from the vertical at the surface of the disk, as a function of cylindrical radius along the disk in the solution of Fig. 1. We can see that, inside the protoplanetary disk, this angle remains everywhere smaller than the nominal minimum value of 30 degrees from the cold outflow analysis of Blandford \& Payne (1982), except near the outer edge of the disk where this angle grows above that value

distortion in the freely-falling region immediately outside. In the distribution of bending angle $\theta$ from the vertical as a function of cylindrical radius along the disk we can see that, inside the protoplanetary disk, this angle remains everywhere smaller than the nominal minimum value of 30 degrees from the cold outflow analysis of Blandford \& Payne (1982), except near the outer edge of the disk where this angle grows above that value (Fig. 3). As we will see in the next section, when matter thermal pressure is taken into account, thermally driven outflows can be launched even for very small values of that angle. However, only in the regions where that angle is high, and at the same time the field line rotation is close to Keplerian and the disk density is high at their footpoint, these outflows will carry the most significant amount of matter. Therefore, although (thermally driven) outflows will be launched from the whole surface of the disk, only the fraction originating around $r_{\text {shock }}$ will lead to the most effective mass outflow rate. Clearly, at radii much larger than $r_{\text {shock, }}$, rotation is insignificant, and the magneto-centrifugal contribution to the wind launching is not sufficient to launch an outflow.

We would like to comment on an important feature of our solution. The physical parameters of the problem enter directly in a combination at the rhs of Eq. (11), and indirectly in the boundary conditions through the disk scale height (which depends on the central mass $m$ and the matter temperature $T$ ). One realizes that, for the same distribution of disk scale height, the magnetic field geometry is independent of the actual physical parameters which enter in the determination of the exact value of the constant in $E q$. (11), since one can always rescale the magnetic field, and therefore obtain the solution of Eq. (11) for a different value of that constant. Of course, its actual value determines how much magnetic flux can be brought in and held inside $r_{\text {shock }}$. As we will see (Eq. (21)), what is important in determining the wind outflow rate, is not the actual magnetic field value, but only its geometry ${ }^{5}$.

\section{The wind outflow}

Having obtained the geometry of field lines in a quasi selfconsistent way, i.e. by assuming current-free conditions in the disk exterior, we will now turn our attention to the study of a possible wind outflow. We will assume that matter below the surface of the disk (as determined in Eq. (4)) remains in almost hydrostatic equilibrium. As we approach the surface layers, if the conditions for the launching of a thermally + magnetically driven outflow are favorable, such an outflow will emerge. It will obviously be highly sub-sonic (in the outward direction) at the surface of the disk, and in direct analogy to the solar wind, it will escape to large distances if and only if it is able to make a smooth sub-sonic to supersonic transition a certain distance above the disk. In order to study the outflow, we will work with the equation of conservation of energy flow along a field/flow line, the Bernoulli equation (e.g. Cao \& Spruit 1994; Kudoh \& Shibata 1997). Safier (1993) showed that, as long as the ionization fraction of the flow exceed $10^{-4}$ (which is true near the surface layers of a protostellar disk where external UV and cosmic ray ionization are important), ambipolar diffusion timescales become much longer than dynamical, and, hence, flux freezing is valid for the outflow. Therefore, once matter emerges above the disk, it is sufficiently well coupled to the magnetic field to justify using ideal MHD in our theoretical treatment of the wind. The above implies that the total energy per unit mass in the corotating frame

$E=\frac{1}{2} v_{\mathrm{p}}^{2}-\frac{1}{2} r^{2} \Omega^{2}-\frac{G m_{\mathrm{d}}}{\left(r^{2}+z^{2}\right)^{1 / 2}}+c_{\mathrm{s}}^{2} \ln \left(\frac{\rho}{\rho_{\mathrm{o}}}\right)$

is a field/flow line constant. Here, $\Omega$ is the "angular rotation rate" of each field line, assumed infinitely rigid. To a good approximation, $\Omega$ is equal to the disk rate of rotation at the distance $r_{\mathrm{o}}$ where the field line is "anchored" along the surface of the disk (Wardle \& Königl 1993), i.e.

$\Omega=\left(\frac{G m_{\mathrm{d}}}{r_{\text {shock }}^{3}}\right)^{1 / 2}\left(\frac{r_{\mathrm{o}}}{r_{\text {shock }}}\right)^{-p}$,

${ }^{5}$ In deriving Eq. (9), we used the relation $\rho_{i} \propto \rho_{\text {disk }}^{\kappa}$ with $\kappa=1 / 2$. This is only strictly valid for densities $<10^{6} \mathrm{~cm}^{-3}$ (Ciolek \& Mouschovias 1994; Ciolek \& Mouschovias 1998). In our problem densities can reach up to $10^{14} \mathrm{~cm}^{-3}$, in which case ionization is very low, with the exponent $\kappa \ll 1$ (Umebayashi \& Nakano 1990). Furthermore, grain collisional friction may be an important effect (Ciolek \& Mouschovias 1993; Ciolek \& Mouschovias 1994). Knowing the limitations of our approximation, we decided to proceed with $\kappa=1 / 2$, since, after all, we are interested in the structure of the outflow which, as we will see, does not depend on the exact value of $\delta$. 


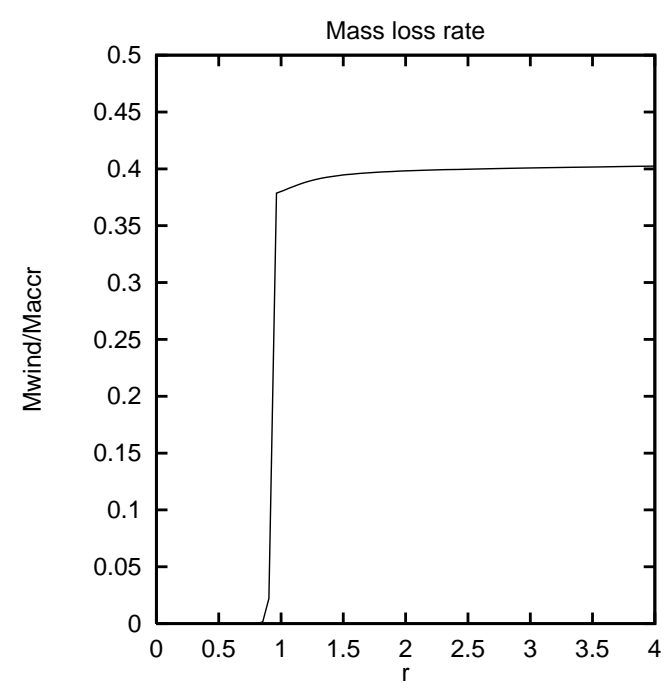

Fig. 4. The distribution of the ratio of mass outflow rate lost in a wind $\dot{m}_{\mathrm{w}}(r)$ to the accretion rate $\dot{m}_{\text {accr }}$, as a function of cylindrical radius $r$ along the disk in the solution of Fig. 1 $\left(\dot{m}_{\mathrm{w}}(r)\right.$ is the total outflow rate originating from disk radii $\leq r)$. Note that the wind outflow originates primarily from a short distance inside $r_{\text {shock }}$

with $p=1$ for $r_{\mathrm{o}} \lesssim r_{\text {shock }}$ (assuming $\left.m\left(r_{\mathrm{o}}\right) \propto r_{\mathrm{o}}\right)$ and $p=2$ for $r_{\mathrm{o}}>r_{\text {shock }}$ (assuming angular momentum conservation in the freely falling exterior). We need to acknowledge at this point that we are not using a self-consistent treatment of gravity, and therefore, our results should not be trusted at radii $r \ll r_{\text {shock }}$.

We define, $r_{\mathrm{o}, \mathrm{s}}, z_{\mathrm{O}, \mathrm{s}}$ and $\rho_{\mathrm{o}, \mathrm{s}}$, as the radius, height and density respectively at the field footpoint on the surface of the disk and at the sonic point along each field line. If we know the position of the sonic point, we can express $E$ at the surface of the disk (where the outflow velocity is assumed to be highly sub-sonic), and at the sonic point, and therefore obtain the matter density at the sonic point as

$$
\begin{aligned}
\rho_{\mathrm{s}}= & \rho_{\mathrm{o}} \exp \left\{-\frac{1}{2}+\frac{\Omega^{2}\left(r_{\mathrm{s}}^{2}-r_{\mathrm{o}}^{2}\right)}{2 c_{\mathrm{s}}^{2}}\right. \\
& \left.-\frac{G m_{\mathrm{d}}}{c_{\mathrm{s}}^{2}}\left(\frac{1}{\left(r_{\mathrm{o}}^{2}+h_{\mathrm{o}}^{2}\right)^{1 / 2}}-\frac{1}{\left(r_{\mathrm{s}}^{2}+z_{\mathrm{s}}^{2}\right)^{1 / 2}}\right)\right\} .
\end{aligned}
$$

Vertical hydrostatic equilibrium implies further that $\rho_{\mathrm{o}}=$ $(2 / \pi)^{1 / 2} \rho_{\text {disk }} \exp (-1 / 2)$ [we have made here the approximation that $\rho(z)=\rho(z=0) \exp \left(-z^{2} /\left[2 h^{2}\right]\right)$, and therefore, $\left.\rho_{\text {disk }}=(\pi / 2)^{1 / 2} \rho(z=0)\right]$. Using the mass conservation condition that $\rho v_{\mathrm{p}} / B_{\mathrm{p}}$ is a field line constant $\left(v_{\mathrm{p}}\right.$ and $B_{\mathrm{p}}$ are the poloidal flow velocity and magnetic field components respectively), we obtain the differential mass outflow rate

$$
\frac{\mathrm{d} \dot{m}_{\mathrm{w}}}{\mathrm{d} \Psi}=2 \cdot \frac{2 \pi \rho_{\mathrm{o}} v_{\mathrm{po}}}{B_{\mathrm{po}}}=\frac{4 \pi \rho_{\mathrm{s}} c_{\mathrm{s}}}{B_{\mathrm{ps}}}
$$

(the extra factor of two takes care of the fact that outflows are launched both above and below the disk). By numerically integrating Eq. (17) across the wind, one can directly calculate the ratio of mass outflow rate lost in a wind to the accretion rate $\dot{m}_{\text {accr }}$ (see Fig. 4 ).
Note that in order to obtain the distribution $\dot{m}_{\mathrm{w}}=$ $\dot{m}_{\mathrm{w}}(r)$, we need to know the distribution of disk density in the disk. Assuming that the mass lost in the wind is only a small percentage of the mass that accretes through the disk allows us to estimate the disk density inside the shock,

$$
\begin{aligned}
& \rho_{\text {disk }}\left(r<r_{\text {shock }}\right)=\rho_{\text {disk }}\left(r_{\text {shock }}^{+}\right) \\
& \times\left(\frac{v_{r}\left(r_{\text {shock }}^{+}\right)}{\dot{r}_{\text {shock }}}\right)\left(\frac{r}{r_{\text {shock }}}\right)^{-2} \\
& =\rho_{\text {disk }}\left(r_{\text {shock }}^{+}\right) \cdot \frac{1}{\omega^{3}}\left(\frac{\dot{m}}{c_{\mathrm{s}}^{3} / G}\right)^{-1}\left(\frac{r}{r_{\text {shock }}}\right)^{-2},
\end{aligned}
$$

and outside the shock,

$$
\begin{aligned}
& \rho_{\text {disk }}\left(r>r_{\text {shock }}\right)=\rho_{\text {disk }}\left(r_{\text {shock }}^{+}\right) \\
& \times\left(\frac{r}{r_{\text {shock }}}\right)^{-2}\left(2-\frac{r_{\text {shock }}}{r}\right)^{-1 / 2}, \text { where }
\end{aligned}
$$

$\rho_{\text {disk }}\left(r_{\text {shock }}^{+}\right)=\frac{\dot{m}}{4 \pi r_{\text {shock }}^{2} c_{\mathrm{s}}}$

$$
=610^{-10} \mathrm{~g} / \mathrm{cm}^{3}
$$

$\times\left(\frac{T}{10 \mathrm{~K}}\right)^{3 / 2}\left(\frac{\dot{m}_{\mathrm{accr}}}{10^{-6} M_{\odot} \mathrm{yr}^{-1}}\right)^{-1}\left(\frac{\omega}{0.1}\right)^{-3}$.

The reader is forewarned that the assumption of negligible mass loss rate in the wind breaks down when $\omega>0.1$, and in that case one should modify the above expressions to take into account the amount of matter lost in the wind along the way. However, our present discussion is already very qualitative, and we thus opted to proceed with the above simple expressions for the disk density.

We have left out for the very end of this section the determination of the position of the sonic point. This is obtained when one takes the derivative of Eq. (14) with respect to the distance $s$ along a field line $\Psi$. One then obtains

$$
\begin{aligned}
\frac{\left(v_{\mathrm{p}}^{2}-c_{\mathrm{s}}^{2}\right)}{v_{\mathrm{p}}} \frac{\mathrm{d} v_{\mathrm{p}}}{\mathrm{d} s}= & {\left[r \Omega^{2}-\frac{G m_{\mathrm{d}}}{\left(r^{2}+z^{2}\right)^{3 / 2}}(r+z t)+\frac{c_{\mathrm{s}}^{2} \alpha}{r}\right] } \\
& \times \frac{1}{\left(1+t^{2}\right)^{1 / 2}} .
\end{aligned}
$$

Here, $t$ is the tangent of the angle the field line makes locally with the horizontal direction; and $\alpha$ is a parameter which characterizes the divergence or convergence of field lines. We have

$\alpha \equiv 1-\frac{\partial \ln t}{\partial \ln r}+\frac{r}{1+t^{2}}\left[\frac{\partial}{\partial r}+t \frac{\partial}{\partial z}\right] \ln t$

(this is obvious if one realizes that $\left[\frac{\partial}{\partial r}+t \frac{\partial}{\partial z}\right]=\frac{\mathrm{d}}{\mathrm{d} s}$ is simply the derivative with the length-path along a given fieldline). $\alpha=1$ for locally parallel field lines, and 2 for a 
Table 1. Percentage of in-flowing material lost in the magnetocentrifugally driven wind originating from a short distance around the centrifugal radius $r_{\text {shock }}$

\begin{tabular}{lccr}
\hline$\omega$ & inside $r_{\text {shock }}$ & outside $r_{\text {shock }}$ & total \\
\hline 0.1 & $1 \%$ & $0.5 \%$ & $1.5 \%$ \\
0.12 & $2 \%$ & $0.5 \%$ & $2.5 \%$ \\
0.14 & $40 \%$ & $0.5 \%$ & $40.5 \%$ \\
0.15 & $55 \%$ & $0.5 \%$ & $55.5 \%$ \\
0.18 & $90 \%$ & $10 \%$ & $100 \%$ \\
$\geq 0.3$ & $80 \%$ & $20 \%$ & $100 \%$ \\
\hline
\end{tabular}

locally monopolar divergence. The last term is negligible if the curvature of the line is small.

Obviously, in order for a wind outflow to make a smooth sonic transition, points $\left(r_{\mathrm{s}}, z_{\mathrm{s}}\right)$ must be found where the right-hand side of Eq. (21) vanishes ${ }^{6}$. These points are obtained numerically, and are plotted as squares in Fig. 1. We emphasize that outflows are generated only along field lines where such points can be found above the surface of the disk.

Some insight on the importance of the physical parameters can be gained by writing the various equations in dimensionless form scaling all distances with $r_{\text {shock }}$ as in the previous section. Thus the ratio of the density at the sonic point to the density at the surface of the disk (Eq. (16)) may be written as,

$$
\begin{aligned}
\frac{\rho_{\mathrm{s}}}{\rho_{\mathrm{o}}}= & \exp \left\{-\frac{1}{2}+\frac{1}{\omega^{2}}\left[\frac{R_{\mathrm{o}}^{-2 p}}{2}\left(R_{\mathrm{s}}^{2}-R_{\mathrm{o}}^{2}\right)\right.\right. \\
& \left.\left.-\frac{1}{\left(R_{\mathrm{o}}^{2}+Z_{\mathrm{o}}^{2}\right)^{1 / 2}}+\frac{1}{\left(R_{\mathrm{s}}^{2}+Z_{\mathrm{s}}^{2}\right)^{1 / 2}}\right]\right\},
\end{aligned}
$$

while the location of the sonic point (rhs of Eq. (21) equals to zero) gives simply,

$R_{\mathrm{S}} R_{\mathrm{o}}^{-2 p}-\frac{R_{\mathrm{s}}+Z_{\mathrm{s}} t}{\left(R_{\mathrm{s}}^{2}+Z_{\mathrm{s}}^{2}\right)^{3 / 2}}+\omega^{2} \frac{\alpha}{R_{\mathrm{s}}}=0$.

Note that $t$ and $\alpha$ are purely geometric factors. As a consequence we see that the location of the sonic point in units of the shock radius and the efficiency of the wind in extracting mass depend on the unique parameter $\omega$. We recall at that point that $\omega$ characterizes the amount of angular momentum remaining in the collapsing core after the initial magnetic braking through torsional Alfvén waves (Basu 1997).

The crucial role of this parameter can be seen in Table 1 , where we summarize the results of our $100 \times 100$ numerical simulations for the ratio $\dot{m}_{\mathrm{w}} / \dot{m}_{\text {accr }}$ for various values of $\omega$. We infer from this table that, when $\omega \lesssim 0.1$ (i.e. when the initial removal of the cloud angular momentum by magnetic braking is very efficient), the mass lost in the

\footnotetext{
${ }^{6}$ The reader should convince him/herself that this is the generalization of the cold wind launching condition discussed in Blandford \& Payne (1982), namely that when $c_{\mathrm{s}}=0$, in order for an accelerated outflow $\left(\mathrm{d} v_{\mathrm{p}} / \mathrm{d} R>0\right)$ to originate from the surface of the disk now at $Z=0$, the condition $t<\sqrt{3}$ needs to be satisfied.
}

wind is a small fraction $(1-2 \%)$ of the amount of matter accreting through the disk. On the other hand, when $\omega>0.1$ (i.e. when the initial removal of the cloud angular momentum by magnetic braking is not very efficient), most of the mass accreting through the disk is eventually lost in a wind outflow originating primarily from a short distance around $r_{\text {shock. }}$. The transition from negligible to dominant outflow takes place over a very small range of $\omega$ values (0.1 to 0.2 ). We offer as food for speculation the idea that an evolution of the value of $\omega$ from small to large values during the collapse allows for the accumulation of only a small amount of the cloud's mass in the inner Keplerian disk, since when $\omega$ grows to a value greater than 0.1 , matter will be lost in a wind instead of accreting to the center.

\section{Discussion}

Our present work derived from an attempt to understand two well-known papers in the study of the magnetic field dragging in accretion disks, Lubow et al. (1994) (hereafter LPP), and Reyes-Ruiz \& Stepinski (1996) (hereafter RS). LPP studied a schematic thin viscous accretion disk, and concluded that, in a flow where both the effective viscosity and effective magnetic diffusivity are due to (magneto-) hydrodynamic turbulent processes, there is hardly any distortion of a magnetic field which originally threads the disk vertically. This implies that the magnetic field remains almost vertical, and consequently, magnetocentrifugally driven outflows cannot be launched from its surface. RS strongly criticized the fact that LPP did not consider the finite disk height, and azimuthal magnetic field in its interior, and went on to incorporate these effects in more physical self-consistent models. Despite their strong criticism, they recover the results of LPP in all their models, except of course in their proto-planetary phenomenological model with no turbulence.

The above works both conclude that, in order to launch magneto-hydrodynamic outflows from the surface of the disk, the magnetic diffusivity must be orders of magnitude smaller than the value obtained if turbulent viscosity is responsible for accretion. There are two ways to achieve this: either ignore turbulence and look for other avenues for accretion, or preferentially (i.e. artificially) suppress the turbulent magnetic diffusivity. Both LPP and RS realized that $\mathcal{P}_{\mathrm{m}} \ll 1$ cannot be physical, and this, together with the observational fact that we do see winds, led them to suggest that protoplanetary disks are non-turbulent and accrete through the removal of angular momentum above (and below) the disk by magnetic torques in a wind. Steady-state solutions where this process seems to work very nicely have been obtained in the literature (Königl 1989; Li 1995; Li 1996; Ferreira \& Pelletier 1995), though a combination of both turbulence and magnetic torque is also another possibility (Casse \& Ferreira 2000).

We on the other hand decided not to ignore the fact that a centrifugally supported disk is turbulent, and to reconsider the original geometry of the magnetic field. 
We then realized the simple fact that, at least in the case of a protoplanetary disk which forms inside an almost freelyfalling protostellar molecular core, the magnetic field outside is highly bent outward (because of the free-fall), and therefore far different from the originally vertical configuration that both LPP and RS considered. This implies that, near the edge of the inner disk, the magnetic field is highly distorted, even without any contribution to that distortion by the disk itself. Such a geometry naturally leads to the generation of thermally + magnetically driven outflows from the surface of the disk, and this is the subject of our present work. We would like to comment that, if we allow for $v_{\mathrm{r}} \neq 0$ in Eq. (5), the field will be distorted slightly more inside the disk, in direct agreement to the results of LPP and RS. We have performed such calculations but decided not to present them since first, the results do not differ much from the case without inner disk accretion, and second, we lack a definite model of inner disk accretion during the Class 0 phase of protostellar collapse.

One could speculate further about the evolution of the inner centrifugally supported disk. Turbulent viscosity will generate internal torques and the disk material will slowly begin to accrete in the form of a standard disk (Shakura \& Sunyaev 1976) provided there is some agent that will remove the excess angular momentum accumulated at the outer edge of that disk. It is interesting that if we relax the working assumption of a current-free magnetosphere, a significant magnetic slowing down torque will be imposed at the footpoints of the magnetic field lines. The torque will be higher in the narrow region around the outer edge of the inner disk, where the magneto-centrifugally driven outflow originates. At this point, the magnetic torque that will develop around $r_{\text {shock }}$ might enter as a "deus ex machina" to remove the excess angular momentum, and thus regulate the overall disk accretion process.

We would like to conclude with a short summary: We have presented a plausible origin for the massive molecular outflows observed at the very early stages of protostar formation (Class 0). The appearance of such an outflow may be an indication for the difference between YSOs of Class 0 and Class 1 YSOs (Lada 1992; André et al. 1993). It is also important to stress that outflows may occur before any central pre-star is formed conversely to the common image of a wind escaping from a disk accreting onto a central object. Most of the mass ejection takes place at the shock interface between the inner centrifugally supported protoplanetary disk, and the outside freely falling molecular cloud core, where the outward bent magnetic field geometry is most favorable to the launching of a magnetocentrifugally driven wind. The wind mass loss rate in our model is controlled by a single parameter $\omega$ which depends on the efficiency of angular momentum loss during the early stages of core collapse. This parameter is likely to be restricted to a narrow range of values, determined by the physics of the early stages of core collapse. If the values of $\omega$ quoted in Basu (1998) are universal (corresponding to very efficient initial removal of the cloud angular momentum by magnetic braking), we may expect an almost constant ratio of ejection to accretion rates of the order of $1 \%$ in YSOs of class 0 . However, a different regime of $\omega$ values exists (corresponding to less efficient initial removal of the cloud angular momentum), where that ratio can be very large (up to $100 \%$ ). This important result should be taken into consideration by observers who try to indirectly infer the core collapse accretion rate from measurements of the molecular wind outflow rate in Class 0 objects (André et al. 1993; Bontemps et al. 1996).

Acknowledgements. We would like to thank Lydie Marchal for her contribution in checking our equations. We acknowledge support from the bilateral scientific agreement Platon between France and Greece. I. C. wishes to acknowledge also partial support by grant 107526 from the General Secretariat of Research and Technology of Greece. He would also like to thank Arieh Königl for introducing him to the problem of magnetic field dragging in accretion disks.

\section{References}

André, P., Ward-Thompson, D., \& Barsony, M. 1993, ApJ, 406, 122

Balbus, S. A., \& Hawley, J. F. 1991, ApJ, 376, 214

Balbus, S. A., Hawley, J. F., \& Stone, J. M. 1996, ApJ, 467, 76

Bardou, A., \& Heyvaerts, J. 1996, A\&A, 307, 1009

Basu, S. 1997, ApJ, 485, 240

Basu, S. 1998, ApJ, 509, 229

Basu, S., \& Mouschovias, T. Ch. 1995a, ApJ, 452, 386 (BM95a)

Basu, S., \& Mouschovias, T. Ch. 1995b, ApJ, 453, 271 (BM95b)

Blandford, R. D., \& Payne, D. G. 1982, MNRAS, 199, 883

Bontemps, S., André, P., Terebey S., \& Cabrit, S. 1996, A\&A, 311,858

Brandenburg, A., Nordlund, A., Stein, R. F., \& Torkelsson, U. 1996, ApJ, 458, 45

Cao, X., \& Spruit, H. C. 1994, A\&A, 287, 80

Casse, F., \& Ferreira, J. 2000, A\&A, 353, 1115

Chandrasekhar, S. 1960, Proc. Natl. Acad. Sci., 46, 253

Chandrasekhar, S. 1981, Hydrodynamic and Hydromagnetic Stability (New York: Dover)

Ciolek, G. E., \& Basu, S. 2000, ApJ, 529, 925

Ciolek, G. E., \& Basu, S. 2001, ApJ, 547, in press

Ciolek, G. E., \& Königl, A. 1998, ApJ, 504, 257 (CK)

Ciolek, G. E., \& Mouschovias, T. Ch. 1993, ApJ, 418, 774

Ciolek, G. E., \& Mouschovias, T. Ch. 1994, ApJ, 425, 142

Ciolek, G. E., \& Mouschovias, T. Ch. 1995, ApJ, 454, 194

Ciolek, G. E., \& Mouschovias, T. Ch. 1998, ApJ, 504, 280

Contopoulos, I., Ciolek, G. E., \& Königl, A. 1998, ApJ, 504, 247 (CCK)

Crutcher, R. M. 1998, in Interstellar Turbulence, Proc. 2nd Guillermo Haro Conf., ed. J. Franco, \& A. Caramiñana (Cambridge)

Crutcher, R. M., Roberts, D. A., Mehringer, D. M., \& Troland, T. H. 1996, ApJ, 462, 79

Dalgarno, A. 1987, in Physical Processes in Interstellar Clouds, ed. G. E. Morfill, \& M. Scholer (Dordrecht: Reidel), 219

Dubrulle, B. 1992, A\&A, 266, 592

Elmegreen, B. G. 1979, ApJ, 232, 729

Ferreira, J., \& Pelletier, G. 1995, A\&A, 295, 807

Foster, P. C., \& Chevalier, R. A. 1993, ApJ, 416, 303

Hayashi, M., Ohashi, N., \& Miyama, S. M. 1993, ApJ, 418, L71

Hunter, C. 1977, ApJ, 218, 834 
Königl, A. 1989, ApJ, 342, 208

Kudoh, T., \& Shibata, K. 1997, ApJ, 474, 362

Lada, C. J. 1992, in The Physics of Star Formation and Early Stellar Evolution, ed. C. J. Lada, \& N. Kylafis, NATO ASI, vol. 342 (Kluwer, Dordrecht), 329

Larson, R. B. 1969, MNRAS, 145, 271

Li, Z.-Y. 1995, ApJ, 444, 848

Li, Z.-Y. 1996, ApJ, 465, 855

Li, Z.-Y. 1998, ApJ, 493, 230

Li, Z.-Y. 1998, ApJ, 497, 850

Li, Z.-Y., \& McKee, C. F. 1996, ApJ, 64, 373

Lubow, S. H., Papaloizou, J. C. B., \& Pringle, J. E. 1994, MNRAS, 267, 235 (LPP)

McDaniel, E. W., \& Mason, E. A. 1973, in The Mobility and Diffusion of Ions and Gases (New York: Wiley)

Mouschovias, T. Ch. 1996, in Solar and Astrophysical Magnetohydrodynamic Flows, ed. K. Tsinganos (Dordrecht: Kluwer), 447

Nakamura, F., Matsumoto, T., Hanawa, T., \& Tomisaka, K. 1999, ApJ, 510, 274

Ohashi, N., Hayashi, M., Ho, P. T. P., \& Momose, M. 1997, ApJ, 475, 211

Ohashi, N., Hayashi, M., Ho, P. T. P., Momose, M., \& Hirano, N. 1996, ApJ, 466, 957
Penston, M. V. 1969, MNRAS, 144, 425

Press, W. H., Flannery, B. P., Teukolsky, S. A., \& Vetterling, W. T. 1988, Numerical Recipes (Cambridge Univ. Press, Cambridge)

Rayleigh, L. 1916, Proc. Roy. Soc. London A, 93, 148

Richard, D., \& Zahn, J.-P. 1999, A\&A, 347, 734

Reyes-Ruiz, M., \& Stepinski, T. F. 1996, ApJ, 459, 653 (RS)

Safier, P. N. 1993, ApJ, 408, 115

Saigo, K., \& Hanawa, T. 1998, ApJ, 493, 342

Saito, M., Kawabe, R., Kitamura, Y., \& Sunada, K. 1996, ApJ, 473,464

Shakura, N. I., \& Sunyaev, R. A. 1976, MNRAS, 175, 613

Shu, F. H. 1977, ApJ, 214, 488

Shu, F. H., \& Li, Z.-Y. 1997 ApJ, 475, 251

Taylor, G. I. 1936a, Proc. Roy. Soc. London A, vol. CLVII, 546

Taylor, G. I. 1936b, Proc. Roy. Soc. London A, vol. CLVII, 565

Umebayashi, T., \& Nakano, T. 1990, MNRAS, 243, 103

Wardle, M., \& Königl, A. 1993, ApJ, 410, 218

Wendt, F. 1933, Ing. Arch., 4, 577

Zahn, J.-P. 1990, Proc. of 6th IAP Astrophysics Meeting/IAU Coll. No 129, ed. C. Bertout, S. Collin-Souffrin, J. P. Lasota, \& J. Tran Thanh Van, 87 